\title{
Acute Kidney Injury as Presenting Symptom of Primary Lymphoma of the Uterus: Uncommon Presentation of a Rare Disease
}

\author{
Alexandra Snyder \\ Inova Fairfax Medical Campus: Inova Fairfax Hospital \\ Ida Dhanuka \\ New York Medical College \\ Haiyan Li \\ Westchester Medical Center \\ Fouzia Shakil \\ Westchester Medical Center \\ Liying Han \\ Westchester Medical Center \\ Sushil Bhardwaj \\ Good Samaritan Hospital Medical Center \\ Gizelka David-West ( $\nabla$ gdavidwestmd@gmail.com ) \\ Westchester Medical Center
}

\section{Case report}

Keywords: lymphoma of uterus, female genital tract lymphoma, acute kidney injury, hydronephrosis

Posted Date: March 22nd, 2021

DOI: https://doi.org/10.21203/rs.3.rs-320928/v1

License: (9) This work is licensed under a Creative Commons Attribution 4.0 International License. Read Full License 


\section{Abstract}

Background: Primary uterine lymphoma is a rare disease, with diffuse large B cell lymphoma being the most common subtype. There are a limited number of reports, series, and reviews in the literature on this disease and its variable clinical presentations. Further data is needed to prevent delay in diagnosis and treatment.

Case: We present the case of a 73-year-old with acute kidney injury secondary to severe bilateral hydronephrosis in setting of an enlarged uterus, confirmed to be primary uterine diffuse large $B$ cell lymphoma.

Conclusion: Acute kidney injury secondary to severe bilateral hydronephrosis is an uncommon clinical presentation of primary uterine lymphoma. This case highlights the importance of heightened awareness of such rare presentations so not to delay diagnosis and treatment of this disease, and emphasizes benefit of surgical resection in the diagnosis and treatment of this disease.

\section{Introduction}

Primary female genital tract lymphoma accounts for $0.2-1.1 \%$ of all extranodal lymphoma cases [1] and less than $1 \%$ of all extranodal non-Hodgkin's lymphoma [2, 3]. Diffuse large B cell lymphoma (DLBCL) is the most common subtype in the female genital tract, and is often found in the ovary, followed by the cervix and then the uterus $[1,4,5]$. Uterine confined DLBCL is the rarest extranodal site of this disease, with approximately 143 reported cases in the English literature [4]. The clinical presentation of primary female genital tract lymphoma is variable and often patients complain of back pain or abnormal vaginal bleeding $[6,7]$. Uncommon but not a unique manifestation of this disease is severe hydronephrosis. We present a case of a post-menopausal woman whose most significant presenting symptom was acute kidney injury secondary to bilateral severe hydronephrosis in the setting of an enlarged uterus.

\section{Case:}

A 73 year-old gravid 0 , para 0 woman with regular gynecologic care and no reported gynecologic history of uterine fibroids, ovarian cysts, or post-menopausal bleeding, presented to her primary care physician for evaluation of progressively worsening back pain and generalized malaise. Baseline laboratories revealed severe acute kidney injury (AKI) and the patient was sent to her local emergency department for further evaluation. Abdominal and pelvic imaging with Computerized tomography (CT) showed an enlarged uterus, severe bilateral hydronephrosis and hydroureter, and free fluid in the pelvis. Pelvic ultrasound showed an enlarged uterus with thickened uterine wall that was hypoechoic and with lobulated irregular contours, and enlarged adnexa bilaterally.

Initial management included placement of percutaneous nephrostomy tubes to alleviate the obstructive AKI, pelvic examination that revealed normal cervix and enlarged uterus and adnexa, followed by endometrial biopsy that was inconclusive and non-diagnostic. She underwent an exploratory laparotomy 
with supracervical abdominal hysterectomy, bilateral salpingoophorectomy, omental biopsy, and cystoscopy. Intra-operative examination revealed an enlarged and homogenously bulky uterus with tumor involving bilateral adnexa, encasing the pelvic ureters bilaterally, and with disease extension to sidewalls bilaterally. Due to extent of disease, the lower uterine segment and cervix were unable to be resected. Cystoscopy was performed with no extension of disease identified.

Histopathologic examination of the uterus, bilateral fallopian tubes, ovaries, and omental biopsy initially on frozen section showed an undifferentiated high-grade lesion. Final pathology of these specimens revealed primary uterine diffuse large B-cell lymphoma, confirmed by infiltration of endometrium with large atypical lymphocytes with immunohistochemistry profile consistent with DLBCL (Fig. 1). Flow cytometry analysis of CD45 positive lymphoid gate was consistent with mature B cell non-Hodgkin lymphoma (Fig. 2). Additional post-operative work up with fluorodeoxyglucose positron emission tomography (FDG-PET)/CT scan showed residual pelvic disease without evidence of distant disease, and bone marrow biopsy and peripheral flow cytometry showed no evidence of lymphoma.

The patient was referred to medical oncology and received 6 cycles of Lenalidomide plus R-CHOP (rituximab, cyclophosphamide, doxorubicin, vincristine) as treatment of her Stage IV, International Prognostic Index [IPI] 4(high), NCCN IPI high to intermediate activated B-cell, large cell, non-Hodgkin's lymphoma. On post-treatment FDG-PET/CT scan, she had complete metabolic response to therapy and complete resolution of hydronephrosis. Her percutaneous nephrostomy tubes were subsequently removed, and she remains without evidence of disease with a disease free interval of 16 months.

\section{Discussion:}

Primary uterine DLBCL is a rare disease, and is the least common of all female genital tract DLBCL $[1,4$, 5]. When DLBCL is found in the uterus, some reports have described it to be confined to the interior of a uterine leiomyoma, making diagnosis challenging due to limitation in tissue sampling $[8,9]$. While endometrial biopsy can be used for diagnosis of primary uterine lymphoma, with limitations as seen in our case, image-guided biopsy is not recommended due to the risk of incorrectly staging a more common ovarian malignancy [1]. Staging of primary uterine DLBCL is often performed by fluorodeoxyglucose positron emission tomography (FDG-PET)/CT due to uterine lymphomas showing increased FDG above physiologic endometrial uptake [2,3].

As highlighted in our case, surgical intervention was required for diagnosis of disease given the insufficient and non-diagnostic endometrial biopsy. However, there is no standardization regarding surgical versus nonsurgical management. There is standardization on treatment regimen, and R-CHOP chemotherapy is considered to be the most accepted and effective regimen [7]. Many of the documented reports of primary uterine and female genital tract DLBCL, including our own, show typical CD20 + markers [10]. This allows for successful R-CHOP treatment with a five year OS and CSS of $70.2 \%$ and $75.2 \%$, and multiple reports of remission $[1,11]$. More recently, a randomized phase 2 trial by Nowakowski 
et al concluded that the addition of Lenalidomide to R-CHOP (R2CHOP) improved progression free survival in newly diagnosed DLBCL [12].

While primary uterine lymphoma usually presents clinically with nonspecific symptoms, post-menopausal uterine bleeding is commonly reported $[1,4,11]$. Other common symptoms include back pain and clinical exam finding of pelvic or abdominal mass $[3,5]$. Although uncommon, hydronephrosis is a documented symptom and presentation of DLBCL of the uterus, cervix, and ovaries. Since hydronephrosis is more often encountered in primary bladder or ureteral malignancy and in squamous cell carcinomas of the gynecologic tract, misdiagnosis or delayed diagnosis of primary DLBCL of the uterus can occur $[6,13]$, making heightened clinical suspicion a priority. The reported cases of hydronephrosis in the literature describe utilization of percutaneous nephrostomy tubes in place of ureteric stents due to tumor extension to the pelvic sidewalls, as was evident in our case [14]. Additionally, other reports describe similar anatomic and pathologic findings as our patient, in which the DLBCL originating in the uterus or uterine cervix had such extensive local invasion that the anatomic boundaries between the uterus, adnexa, bladder, rectum, and inferior vaginal wall were compromised, leading to incompletely resected disease [3, 15]. Our case, and others, highlight the benefit of surgical resection for diagnosis of disease with demonstration of successful response to medical therapy and clinical remission even with residual disease at time of surgical resection.

\section{Conclusion}

Primary uterine DLBCL is the rarest of all primary genital tract lymphomas, with variable clinical presentation of disease. Diagnosis of this disease also proves challenging due to the limitations of tissue sampling via endometrial biopsy and percutaneous image-guided biopsy. Our case emphasizes the uncommon presentation of AKI secondary to severe hydronephrosis and the importance of heightened clinical suspicion for this disease, especially in a patient with no reported abnormal gynecologic history, presenting with symptoms and clinical exam findings not typical of more common uterine cancers. Our case also highlights the benefit of surgical intervention for diagnosis of disease, and the successful treatment with R2CHOP leading to clinical remission even with residual and unresected disease.

\section{Declarations}

\section{Declarations}

Ethics approval and consent to participate: Ethics and IRB approval is not required at our institution for the publication of case reports. Consent for publication was obtained from patient.

Consent for publication: Consent was obtained from patient and is on file upon request.

Availability of data and materials: Not applicable to case report

Competing Interests: The authors declare that they have no competing interests. 
Funding: The authors have no funding sources

Authors' Contributions: All authors read and approved the final manuscript. AS reviewed patient records and wrote first draft of manuscript. ID reviewed patient records and wrote first draft of manuscript. HL performed grossing of pathologic specimen and prepared pathologic imaging contributions to the manuscript. FS oversaw the grossing of the pathologic specimen, provided the final report of the pathologic diagnosis, and reviewed pathology imaging contributions to the manuscript. LH oversaw the grossing of the pathologic specimen and reviewed pathology imaging contributions to the manuscript. SB participated in the treatment and management of the patient and was involved in review and editing of the manuscript. GD participated in the treatment and management of the patient, contributed to writing the manuscript, performed final review and edits of the manuscript, and organized submission of the manuscript for publication.

Acknowledgements: Not applicable

\section{References}

1. Nasioudis D, Kampaktsis PN, Frey M, et al. Primary lymphoma of the female.

2. genital tract. An analysis of 697 cases. Gynecol Oncol. 2017;145(2):305-9. doi:10.1016/j.ygyno.2017.02.043.

3. Gong J, Dong A, Wang Y, et al. Primary Uterine Peripheral T-cell Lymphoma. Medicine. 2016;95(17). doi:10.1097/md.0000000000003532.

4. Chen B-A, Chen R, Yu Z, Zhang H, Ding J. Primary malignant lymphoma of the uterus and broad ligament: a case report and review of literature. OncoTargets Therapy. 2015:265. doi:10.2147/ott.s78171.

5. Mandato VD, Palermo R, Falbo A, et al. Primary Diffuse Large B-Cell Lymphoma of the Uterus: Case Report and Review. Anticancer Res. 2014;34:4377-90.

6. Wang J, Zeng L, Chen S, et al. Lymphoma of the female genital tract: a clinicopatholngical analysis of 25 cases. American Journal of Translational Research. 2019;11(9):5800-11. doi:19438141/AJTR0100358.

7. Chen $P$, Jiang $M$, Lin $Y, Y e X$, Ruan X, Huang Q. Primary diffuse large B-cell lymphoma of the left ureter: A case report. Molecular Clinical Oncology. 2016;5(3):255-7. doi:10.3892/mco.2016.949.

8. Ishii Y, Fujisawa S, Ando T, et al. Primary uterine lymphoma: The Yokohama Cooperative Study Group for Hematology (YACHT) study. Asia-Pac J Clin Oncol. 2018;14:e455-9. https://doi.org/10.1111/ajco.1304.

9. Zhao L, Ma Q, Wang Q, Zeng Y, Luo Q, Xiao H. Primary diffuse large B cell lymphoma arising from a leiomyoma of the uterine corpus. Diagn Pathol. 2016;11(1). doi:10.1186/s13000-016-0464-8.

10. Martin A, Medlin E. Primary, non-germinal center, double-expressor diffuse large B cell lymphoma confined to a uterine leiomyoma: A case report. Gynecologic Oncology Reports. 2017;22:78-81. 
doi:10.1016/j.gore.2017.10.003.

11. Vang R, Medeiros LJ, Ha CS, Deavers M. Non-Hodgkins Lymphomas Involving the Uterus: A Clinicopathologic Analysis of 26 Cases. Mod Pathol. 2000;13(1):19-28. doi:10.1038/modpathol.3880005.

12. Frey NV, Svoboda J, Andreadis $C$, et al. Primary lymphomas of the cervix and uterus: The University of Pennsylvania's experience and a review of the literature. Leuk Lymphoma. 2006;47(9):1894-901. doi:10.1080/10428190600687653.

13. Nowakowski GS, Hong F, Scott DW, et al. Addition of lenalidomide to R-CHOP (R2CHOP) improves outcomes in newly diagnosed diffuse large B-cell lymphoma (DLBCL): first report of ECOGACRIN1412 a randomized phase 2 US intergroup study of R2CHOP VS R-CHOP. Hematological Oncology. June 2019; https://doi.org/10.1002/hon.6_2629.

14. Kawaguchi Y, Kimura S, Momozono K, Igawa T, Noguchi M. Malignant lymphoma of the bladder with bilateral hydronephrosis. Rare Tumors. 2019;11:203636131882516. doi:10.1177/2036361318825165.

15. Samama M, Poelgeest MV. Primary Malignant Lymphoma of the Uterus: A Case Report and Review of the Literature. Case Reports in Oncology. 2011;4(3):560-3. doi:10.1159/000334852.

16. Cubo AM, Soto ZM, Cruz M, et al. Primary diffuse large B cell lymphoma of the uterine cervix successfully treated by combined chemotherapy alone. Medicine. 2017;96(19). doi:10.1097/md.0000000000006846.

\section{Figures}




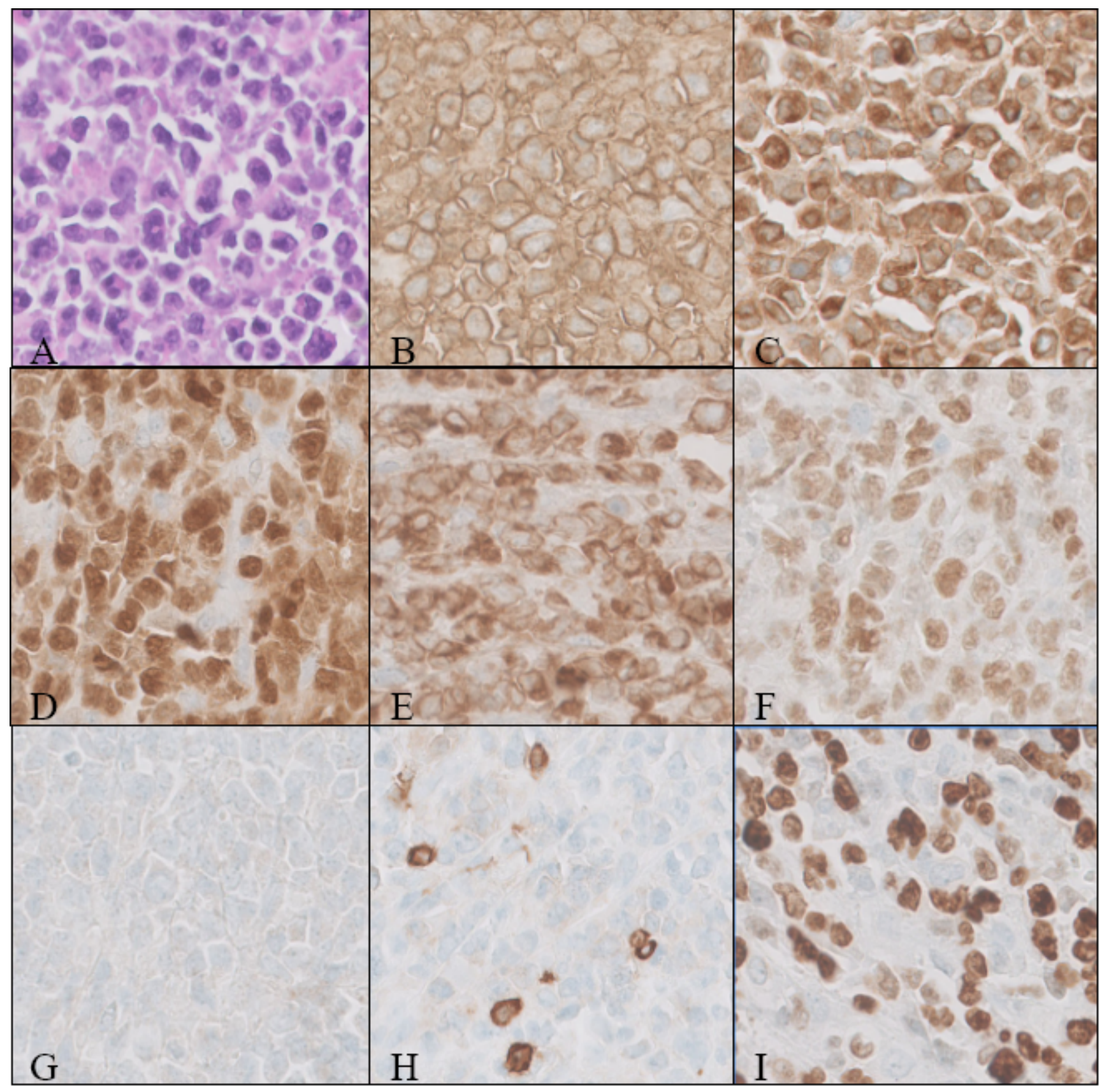

\section{Figure 1}

Histological and immunohistochemical analysis of uterine endometrium shows: A) The endometrium is diffusely infiltrated by large atypical lymphocytes. The lymphocytes have high nuclear to cytoplasmic ratio, clumped to vesicular chromatin and prominent nucleoli (H\&E); B) The malignant lymphocytes are diffusely strong stained by CD20; C) The malignant lymphocytes are diffusely stained by CD79a; D) The nuclei of the malignant lymphocytes are positive for MUM-1; E) The malignant lymphocytes are also positive for Bcl-2; F) The malignant lymphocytes are weakly and partially stained by $\mathrm{Bcl}-6 ; \mathrm{G}$ ) The malignant lymphocytes are negative for $\mathrm{CD} 10 ; \mathrm{H}$ ) The malignant lymphocytes are negative for $\mathrm{CD} 5$; the scattered T cells are highlighted by CD5; I) Ki-67 shows a proliferative index of $70-80 \%$ of the large malignant lymphocytes. (A-I: original magnification $\times 400$ ) 


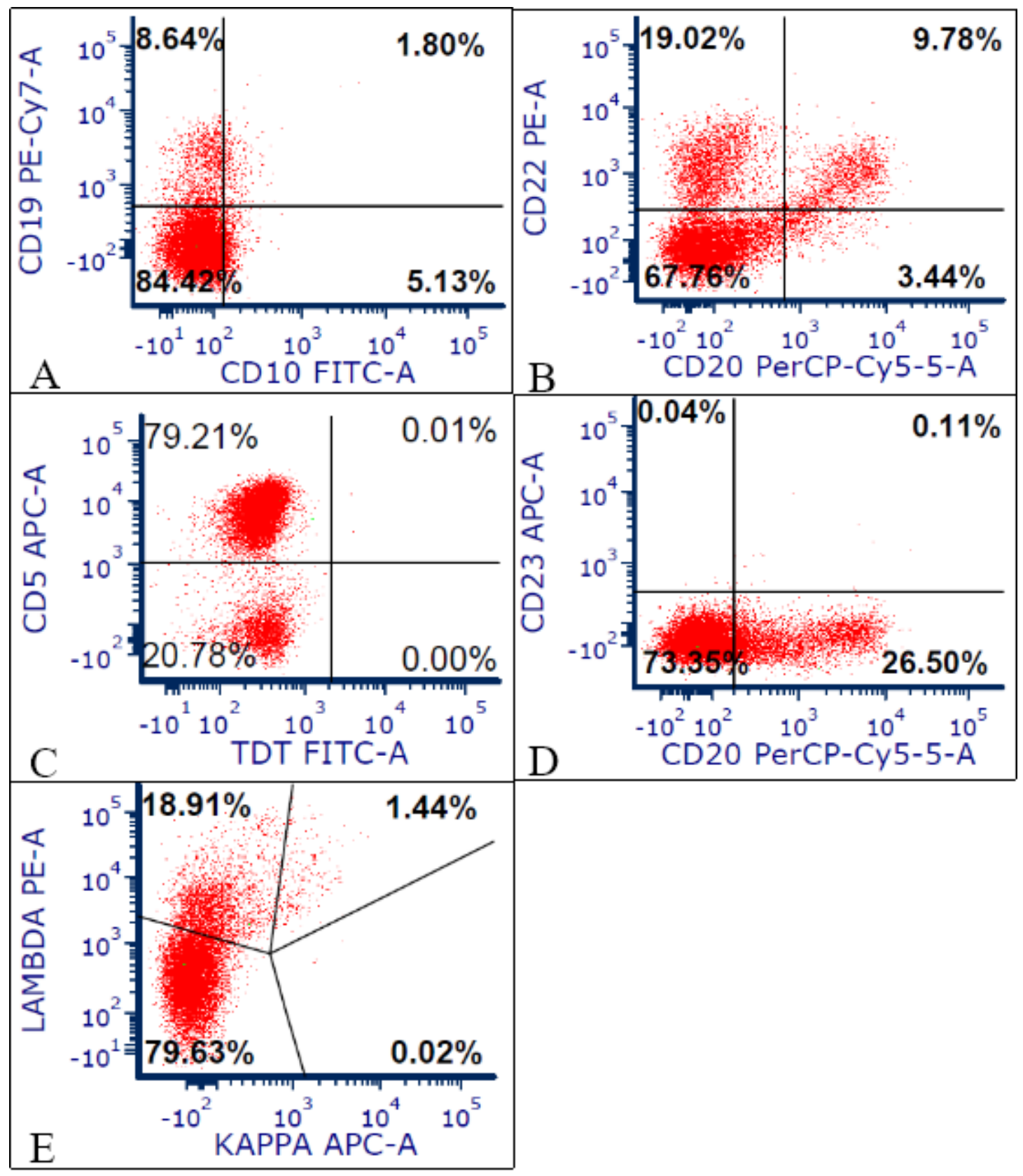

Figure 2

Flow cytometry analysis of CD45 positive lymphoid gate reveals an abnormal population of BLymphocytes. A) The cells are positive for CD19 and CD10 (dim) (left upper quadrant; B) The cells are positive for CD20 and CD 22 (right upper quadrant); C) The cells are negative for Tdt and CD5 (left upper quadrant); D) The cells are negative for $\mathrm{CD} 23$ (right lower quadrant); E) The cells are surface lambda light chain restricted and not expressing kappa light chain on the cell surface (left upper quadrant). 